\title{
Advance care planning discussions among residents of long term care and designated assisted living: experience from Calgary, Alberta
}

\author{
Claire Dyason, ${ }^{1}$ Jessica Simon, ${ }^{2,3}$ Tracy Lynn Wityk Martin ${ }^{1}$
}

- Additional material is published online only. To view please visit the journal online (http://dx.doi.org/10.1136/ bmjspcare-2013-000444).

${ }^{1}$ Palliative/End of Life Care, Alberta Health Services, Calgary Zone, Calgary, Alberta, Canada ${ }^{2}$ Advance Care Planning and Goals of Care Program, Alberta Health Services, Calgary Zone, Calgary, Alberta, Canada ${ }^{3}$ Division of Palliative Medicine, Department of Oncology, University of Calgary, Calgary, Alberta, Canada

\section{Correspondence to}

Dr Jessica Simon, 710 South Tower, 140329 St NW, Calgary, Alberta, Canada T2N 2T9; jessica.simon@ albertahealthservices.ca

Received 5 January 2013 Revised 17 June 2013 Accepted 9 September 2013 Published Online First 25 November 2013

\section{CrossMark}

To cite: Dyason C, Simon J, Wityk Martin TL. BMJ Supportive \& Palliative Care 2015;5:48-53.

\section{ABSTRACT}

Objectives Patients, physicians and the healthcare system are faced with the challenge of determining, and respecting, the medical wishes of an aging population. Our study sought to describe who participates in advance care planning (ACP) and decision-making for patients in long-term care and designated assisted living. Methods In 2008, Alberta Health Services initiated its 'Advance Care Planning: Goals of Care Designation' (Adult) policy in the Calgary zone. This policy encouraged discussions about goals of care and used a tracking form to capture these conversations. A postpolicy implementation chart review was performed at 3 time points: at baseline, at 6 months and at 18 months post implementation in long term care (LTC) and designated assisted living sites.

Results 166 charts were reviewed and 90\% had a documented goals of care order. Less than half of residents (47\%) were documented as participating in conversations and they were less likely to participate if they had cognitive impairment and were living in LTC. Documented family participation was more prevalent in LTC ( $51 \%$ vs $11 \%$ ). Nurses participated in $67 \%$ of documented conversations with only $34 \%$ of discussions documenting physician involvement.

Conclusions This study identifies the lack of documented resident participation in ACP in LTC. While this finding may be explained by the high prevalence of cognitive impairment in our population, it raises questions about the optimal approach to ACP in LTC. In this setting, ACP appears to be more about relational autonomy than it is about patient autonomy.

\section{INTRODUCTION}

The percentage of the Canadian population over the age of 65 years is expected to increase from $11.6 \%$ in 1993 to $23 \%$ in $2041 .^{1}$ As of $2009-2010$, there were
4633 residential care facilities in Canada with 274270 residents; this number will also rise in the coming decades. ${ }^{2}$ Patients, families, physicians and the healthcare system as a whole are faced with the difficult challenge of determining and respecting, the medical wishes of this aging and increasingly dependent population. Advance care planning (ACP) is part of the response to this challenge. ${ }^{3}$ Without a thorough understanding and discussion of a person's wishes related to healthcare, physicians, patients and their family members may find themselves in conflict, and providing or receiving unwanted care. ${ }^{4}$

An American study interviewing family members of individuals who had recently died found that $70 \%$ of individuals had an advance directive and that these people were more likely to have been women, older and Caucasian. ${ }^{5}$ These individuals also were less likely to die in the intensive care unit, on a respirator or to have had a feeding tube in the last month of life. ${ }^{5}$ Another American survey found that $43 \%$ of individuals who died between 2000 and 2006 had required medical decision making in the last days of life and $70 \%$ of these individuals had not had decision-making capacity. ${ }^{6}$ Factors that predicted the loss of decision-making capacity included cognitive impairment, residence in a nursing home and cerebrovascular disease. ${ }^{6}$

In the Calgary Health Region, individuals requiring residential support may reside in one of two levels of care: long term care (LTC) or designated assisted living (DAL). LTC (similar to 'Nursing care home' or a skilled nursing facility) provides care for individuals who have 
complex medical needs requiring 24-hour onsite registered nurse assessment and treatment as well as regularly scheduled and unscheduled onsite physician support. ${ }^{7}$ DAL (similar to 'personal care home') provides scheduled and unscheduled personal care and 24 h support by Licensed Practical Nurses and Health Care Aides. They do not need the services of an onsite registered nurse. ${ }^{7}$ Residents of both settings are required to pay accommodation fees and are responsible for furnishings, personal effects and copayments for medications and medical equipment. Both these entities receive some public funds through provincial medical insurance and are commonly privately delivered.

In November 2008, Calgary Health Region (now Alberta Health Services (AHS), Calgary zone) implemented an Advance Care Planning: Goals of Care Designation (Adult) (ACP:GCD) policy $^{8}$ across all healthcare sectors, including acute care facilities, emergency departments, LTC, DAL and hospices. Prior to the ACP:GCD policy introduction, 'do not resuscitate' and/ or 'levels of care' orders were used within the health region. These primarily described use or withholding of cardiopulmonary resuscitation. The number of levels and descriptions differed between sectors. There was no standard approach to the communication or documentation of ACP discussions across the region.

The intent of the policy was to encourage communication about decision making. It provided a framework for efficient communication of the general intent of care through goals of care designations (GCD). These describe resuscitative, medical or comfort care providing direction on specific interventions and locations of care (see online supplementary appendix 1). Healthcare providers were instructed to discuss six elements with patients and families and record them on a tracking record (see online supplementary appendix 2). These included: prognosis and anticipated outcomes, patients' values and understanding of treatment options, life sustaining measures, comfort measures, resources available to them and finally GCD order discussion. The policy required residents of LTC to have GCDs within 45 days of admission and residents of DAL to have GCDs within 90 days of admission through a process of ACP.

Our study analysed the data obtained as part of an evaluation and quality improvement (QI) audit of AHS' ACP:GCD (Adult) policy to better understand who participated in advance care decision making for residents in LTC and DAL and which factors such as cognitive status or location of care may influence this process.

\section{METHODS}

Data was collected as part of the evaluation and QI of this policy implementation and included a chart audit that was performed at three time points: at baseline, at 6 months and at 18 months after policy implementation. Data were collected by experienced data coordinators with a nursing background, through a review of the charts of 166 randomly selected residents. Calgary Health Region Quality, Safety and Health Improvement (which is now Data Integration Measurement and Reporting within AHS) reviewed Electronic Health Record data and provided lists of charts that met sampling criteria. Chart numbers were randomly sorted in an Excel spreadsheet and were reviewed by the audit team in sorted order until sample size was met. Sample size was determined based on data needs and feasibility for the evaluation/ QI work, aiming for either $10 \%$ of the sample population or $\mathrm{n}=30$ whichever was greater at each site (not always achieved). Data was obtained predominately from the 'ACP Tracking Record' (see online supplementary appendix 2). This document was created as part of the policy to record ACP conversations across sectors and included discussion content and a list of participants. In addition, the charts were reviewed for other data including demographics, the GCD order and for any additional documentation of participants in ACP conversation (outside of the ACP Tracking Record).

Residents were included in the chart review if they had a minimum length of stay of 45 days in LTC or 90 days in DAL and if they were older than 50 years of age. Five LTC sites including one rural LTC site and two DAL sites participated in the evaluation/QI. LTC sites were selected for size (four largest urban sites were selected to be most representative of population) and prior relationship with the ACP:GCD programme (one rural site). To reflect various experiences with policy implementation, one DAL site with perceived challenges was selected, and one DAL site with dedicated ACP:GCD resources was selected. For each chart reviewed, up to five ACP conversations (almost all of which had occurred in the LTC or DAL) were audited. General demographics were collected as well as information on resident diagnosis, cognitive status as included under medical diagnoses in the chart such as dementia, memory impairment and cognitive impairment, and current GCD. As well, data was examined to see whether residents, family members or healthcare professionals were documented as participating in these conversations. To reflect the more established process, we analysed data from 6 months and 18 months postpolicy implementation in this study. This study excluded charts with no documentation of ACP, as this study was focused on analysing who had participated in ACP in LTC and DAL in the former Calgary Health Region.

\section{RESULTS}

In total, 200 charts were reviewed in the chart audit; of these, 166 charts had documented ACP conversations $(83 \%)$. Only data from those 166 were reviewed 
Table 1 GCD orders in LTC/DAL at 6 months and 18 months

\begin{tabular}{lllll}
\hline & \multicolumn{2}{c}{6 months time point } & \multicolumn{2}{c}{18 months time point } \\
\hline GCD & DAL & LTC & DAL & LTC \\
R1 & $4(15 \%)$ & $6(13 \%)$ & $7(27 \%)$ & $5(7 \%)$ \\
R2 & 0 & 0 & $1(4 \%)$ & $3(4 \%)$ \\
R3 & $5(19 \%)$ & $2(4 \%)$ & $4(15 \%)$ & $1(2 \%)$ \\
M1 & $4(15 \%)$ & $14(30 \%)$ & $12(46 \%)$ & $26(39 \%)$ \\
M2 & $1(4 \%)$ & $9(19 \%)$ & $1(4 \%)$ & $14(21 \%)$ \\
C1 & $2(8 \%)$ & $9(19 \%)$ & 0 & $15(22 \%)$ \\
C2 & $1(4 \%)$ & $3(6 \%)$ & 0 & $1(2 \%)$ \\
Not documented & $9(35 \%)$ & $4(9 \%)$ & $1(4 \%)$ & $2(3 \%)$ \\
\hline
\end{tabular}

DAL, designated assisted living; GCD, goal of care designations; LTC, long term care (supplementary appendix 1 describes each GCD).

in this study, as it focused on ACP conversations. Of these, 114 charts were from LTC and 52 were from DAL facilities. The majority of residents were female (64\%). The largest age group was aged between $85-94$ years (43\%), while $36 \%$ were between the ages of $75-84$ years old. The prevalence of cognitive impairment was $78 \%$ among residents in LTC and 46\% among patients in DAL. In total there were 272 ACP conversations documented in the 166 reviewed charts.

Among these charts, 150 (90\%) had a documented GCD order in the chart. Resuscitative care orders (including some form of cardiopulmonary resuscitation, intubation or admission to intensive care) were present for $40 \%$ (21) of individuals who were living in DAL. Another 35\% wished to receive medical care to prolong life, to be transferred to hospital but not to receive any resuscitative care and only $6 \%$ wished for comfort care. In long-term care, only 15\% (17) of residents had orders for resuscitative care while the majority (55\%) had orders for life-prolonging care treatments excluding resuscitation and $25 \%$ had orders for comfort care. Table 1 provides information on GCD at 6 months and 18 months.

Residents were documented to be present in conversations about their own wishes $47 \%$ of the time, while they were documented as not participating $41 \%$ of the time. In $12 \%$ of these conversations it was not documented if residents were present or not. Many more family members participated in these conversations with $62 \%$ of discussions having a documented family member present versus 28\% not present and 9\% not documented in the chart. Table 2 shows resident and family participation in reference to cognitive impairment and the location of care. Residents with cognitive impairment were less likely to be present, with only $20 \%$ documented as present at ACP conversations. Family participation increased when patients had cognitive impairment, with $47 \%$ of these residents having family documented as present at these conversations. However $6 \%$ of patients who were not documented as cognitively impaired were not present at these conversations.

When we further examined this data and stratified for location of care (DAL vs LTC), we found that $55 \%$ of the time documentation reflected that residents in DAL were unaccompanied in these conversations; however in LTC it was only $12 \%$ (see figure 1 ).

Healthcare provider participation also varied depending on location of care. Overall, nurses were the most commonly represented group, with documented presence in $67 \%$ of conversations. Physicians were only recorded present $34 \%$ of the time $(16 \%$ in DAL, $43 \%$ in LTC) along with social workers $16 \%$, spiritual care $2 \%$ and others including pharmacists, physical and occupational therapists $15 \%$ of the time.

When we stratified for multiple healthcare providers and location of care we found the presence of interdisciplinary teams in LTC with $21 \%$ of conversations having a nurse, physician and another healthcare provider present. This was not a feature in conversations that occurred in DAL, with only $10 \%$ having a nurse and another healthcare provider present and 2\% with a nurse and physician present (figure 2).

\section{DISCUSSION}

The aim of our study was to establish who participated in ACP conversations in care facilities in Calgary after the implementation of the ACP:GCD policy. Of concern, we found that less than half of all charts contained documentation that the resident had been present for his or her own ACP conversation. The ACP:GCD (Adult) policy emphasises the

Table 2 Role of cognitive impairment in resident and patient participation

\begin{tabular}{|c|c|c|c|c|c|c|c|}
\hline \multirow[b]{2}{*}{ Cognitive impairment } & \multicolumn{3}{|c|}{ Resident participation } & \multicolumn{3}{|c|}{ Family participation } & \multirow[b]{2}{*}{ Total discussions } \\
\hline & Yes & No & Not doc & Yes & No & Not doc & \\
\hline$\overline{\mathrm{DAL}}$ & & & & & & & 91 \\
\hline Yes & $25(27 \%)$ & $9(10 \%)$ & $4(4 \%)$ & $20(22 \%)$ & $15(16 \%)$ & $3(3 \%)$ & \\
\hline No & $36(40 \%)$ & $2(2 \%)$ & $1(1 \%)$ & $7(8 \%)$ & $25(27 \%)$ & 0 & \\
\hline LTC & & & & & & & 182 \\
\hline Yes & $31(17 \%)$ & $85(47 \%)$ & $26(14 \%)$ & $108(59 \%)$ & $15(8 \%)$ & $18(10 \%)$ & \\
\hline No & $23(13 \%)$ & $15(8 \%)$ & $14(8 \%)$ & $23(13 \%)$ & $13(7 \%)$ & $5(3 \%)$ & \\
\hline
\end{tabular}

DAL, designated assisted living; LTC, long term care. 


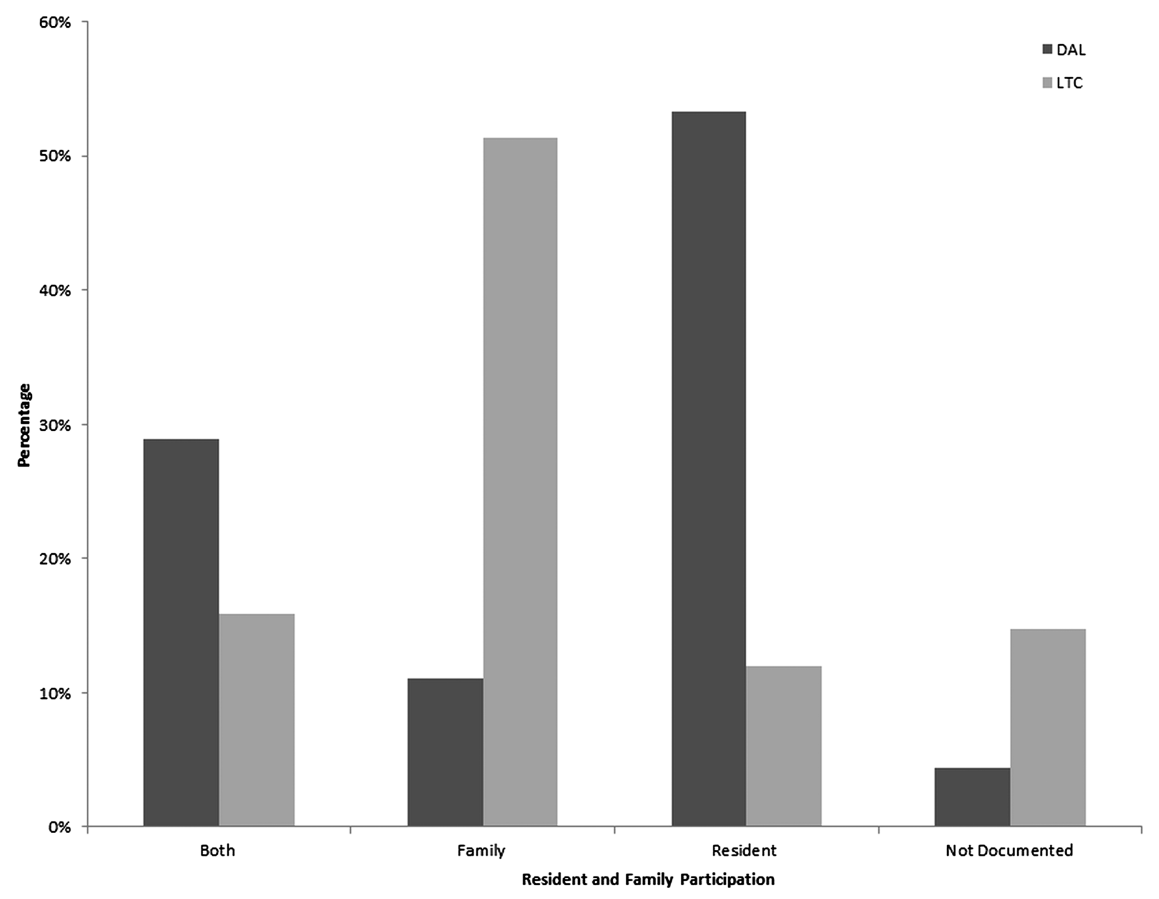

Figure 1 Resident and family participation. DAL, designated assisted living; LTC, long term care.

involvement of the individual primarily and to use surrogate decision makers only when necessary. It is understandable that residents with cognitive impairment were less likely to participate in ACP discussions and were more likely to have family present at these conversations. The severity of cognitive impairment plays a role in determining whether residents have capacity to participate in ACP conversations.

Although we were unable to quantify the degree of cognitive impairment through our chart review, this

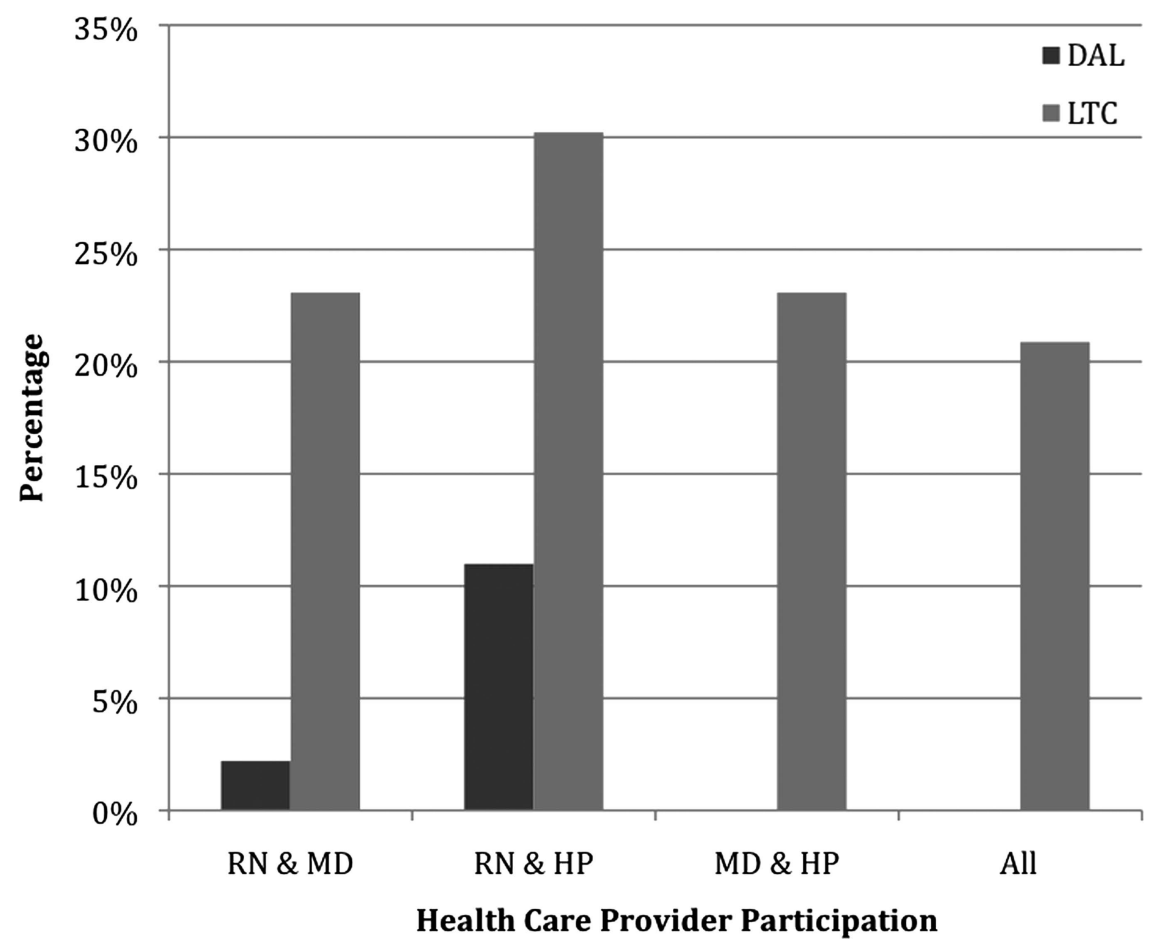

Figure 2 Healthcare provider participation. DAL, designated assisted living; HP, allied healthcare provider; LTC, long term care; MD, medical doctor; RN, registered nurse. 
would certainly be an area that deserves more study. We could also not assess whether a prior advance directive was used to guide decision making during these conversations. Ideally, ACP should be occurring early in a person's disease (before they require residential support) so as to maximise the likelihood and ability of the person to participate. ${ }^{9}$ While the ACP:GCD policy applies to primary care settings as well as hospitals, LTC, DAL, emergency departments, home care and residential hospices, there were no 'standards' or 'timing' recommendations for adherence to the policy in the primary care setting. It would be beneficial to explore whether implementation standards in primary care could increase engagement in ACP prior to or early in illness, particularly cognitive impairment.

Family presence in planning conversations was common even in the absence of cognitive impairment, particularly in LTC. Nolan et al ${ }^{10}$ examined the preferences of patients with terminal diagnoses regarding the inclusion of their family members and physicians in decision making. If they were capable of decision making, half of the patients wished to be the sole decision maker and $44 \%$ wished to have shared decision making with family. Another study of the perspectives of American community-dwelling individuals, aged 70 years and above, on surrogate decision making, found that $95 \%$ of individuals had someone who they trusted to make decisions for them if they became incapable but only $49 \%$ of them had discussed their preferences with these people and only 9\% had a living will. ${ }^{11}$ The relationship between engaging in ACP and decisional congruence has been well explored. ${ }^{3}$ This has implications for ACP in LTC as our study shows that many residents may be relying on family members to make these decions. This brings up ethical and practical concerns surrounding how we best prepare and support families in this process and continue to honour patient values.

In Calgary, there was a more established process for accessing physicians and interdisciplinary teams in LTC than in DAL. This may explain the greater use of teams in these conversations in LTC. Physicians, nurse practitioners and nurses have been shown to be effective for engaging in ACP with patients ${ }^{12}{ }^{13}$ but little is known about the impact of team versus individual healthcare provider participation in ACP discussions.

There are several inherent limitations to this study. It used retrospective data collected for the purpose of a policy evaluation/QI audit. Moreover, it relies on the quality and the accuracy of the original documentation on the tracking record. It cannot answer important questions such as optimal timing of ACP after admission to a care facility. Nor can it assess the impact of who was present on outcomes for the resident, family, staff and healthcare system. The strength of the study is that it provides information on the 'real-life' context of ACP conversations and GCD determinations in care facilities. The data suggests that the communication occurring in LTC, and to a lesser degree in DAL, was not actually 'advance' care planning by residents themselves but was often surrogate care planning and decision making related to GCD. Relational autonomy ${ }^{14}{ }^{15}$ rather than absolute patient autonomy is therefore predominant within this process, particularly as nursing care needs increase. Finally the data highlight the importance of engaging in ACP while one is healthy and that those who delay participating in ACP until they 'get sick' or are admitted to residential care facilities will often have missed out on the opportunity to participate in decisions about their own care or to guide their families and healthcare professionals in those decisions.

Acknowledgements We would like to thank: Bev Berg, Manager Advance Care Planning/Goals of Care and The Grief Support Program, Palliative/End of Life Care, Alberta Health Services, Calgary Zone and the rest of the Care at the End of Life Initiative Team from the former Calgary Health Region Team and current ACP:GCD Team, Calgary Zone, Alberta Health Services.

Competing interests None.

Ethics approval Research ethics board approval was obtained through the local Conjoint Health Research Ethics Board to analyse and publish this QI data. Data was analysed using descriptive statistics on Excel.

Provenance and peer review Not commissioned; externally peer reviewed.

\section{REFERENCES}

1 Chertkow H. Diagnosis and treatment of dementia: introduction. CMAJ 2008;178:316-20.

2 Statistics Canada. Residential Care Facilites, Catalogue no.83-237-X, 2009-2010, 8. 2011.

3 Jordens C, Little M, Kerridge I, et al. From advance directives to advance care planning: current legal status, ethical rationales and a new research agenda. Intern Med J 2005;35: 563-6.

4 Detering KM, Hancock AD, Reade MC, et al. The Impact of advance care planning on end of life care in elderly patients: randomized controlled trial. BMJ 2010;340:c1345.

5 Teno JM, Gruneir A, Schwartz Z, et al. Association between advance directives and quality end-of-life care: a national study. JAGS 2007;55:189-94.

6 Silveira MJ, Kim SYH, Langa KM. Advance directives and outcomes of surrogate decision making before death. NEJM 2010;362:1211-18.

7 Alberta Health Services. Admission Guidelines of Publicly Funded Continuing Care Living Options. 2010. http://www. albertahealthservices.ca/Seniors/if-sen-living-option-guidelines. pdf (accessed 10 Jun 2013).

8 Advance Care Planning-Goals of Care; Alberta Health Services. 2012. http://www.albertahealthservices.ca/advancecareplanning. asp (accessed 23 Dec 2012).

9 Herrmann N, Gauthier S. Diagnosis and treatment of dementia: 6. Management of severe Alzheimer disease. CMAJ 2008;179:1279-87.

10 Nolan MT, Hughes M, Narendra DP, et al. When patients lack capacity: the roles that patients with terminal diagnosis would choose for their physicians and loved ones in making medical decisions. J Pain Symptom Manage 2005;30: $342-50$. 
11 Hopp F. Preferences for surrogate decision makers, informal communication and advance directives among communitydwelling elders: results from a national study. Gerontologist 2000;40:449-57.

12 Lawrence JF. The advance directive prevalence in long-term care: a comparison of relationships between nurse practitioner health care model and a traditional health care model. J Am Acad Nurse Pract 2009;21:179-85.
13 Jeong SY, Higgins I, McMillan M. Advance Care Planning (ACP): the nurse as 'broker' in residential aged care facilities. Contemp Nurse 2007;26:184-95.

14 Robinson CA. Advance care planning: re-visioning our ethical approach. Can J Nurs Res 2011;43:18-37.

15 Ho A. Relational autonomy or undue pressure? Family's role in medical decision making. Scand J Caring Sci 2008;22: 128-35. 